\title{
The three-dimensional pair distribution function analysis of disordered single crystals Basic concepts
}

\author{
Journal Article \\ Author(s): \\ Weber, Thomas; Simonov, Arkadiy (i) \\ Publication date: \\ 2012 \\ Permanent link: \\ https://doi.org/10.3929/ethz-b-000049761 \\ Rights / license: \\ In Copyright - Non-Commercial Use Permitted \\ Originally published in: \\ Zeitschrift für Kristallographie 227(5), https://doi.org/10.1524/zkri.2012.1504
}




\title{
The three-dimensional pair distribution function analysis of disordered single crystals: basic concepts
}

\author{
Thomas Weber* and Arkadiy Simonov \\ Laboratory of Crystallography, ETH Zurich Wolfgang-Pauli-Str. 10, 8093 Zurich, Switzerland
}

Received December 9, 2011; accepted January 23, 2012

\section{D-Pair distribution function / \\ Pair distribution function analysis / Disorder / \\ Diffuse scattering}

\begin{abstract}
Theory and principles of the three-dimensional pair distribution function analysis of disordered single crystals are introduced. The mathematical framework is presented and the appearance of pair distribution function patterns is discussed on the examples of some basic disorder models. It is further demonstrated how pair distribution function maps are affected by typical experimental problems. Approaches for a better understanding of such effects and strategies for a proper handling of artifacts in diffuse scattering experiments are proposed.
\end{abstract}

\section{Introduction}

In the recent years the pair distribution function analysis of powder samples (powder PDF) has become a popular tool for investigating disordered structures. The powder PDF is the Fourier transform of the total X-ray or neutron powder diffraction pattern of a sample and provides a direct measure for the real interatomic distances $|\mathbf{r}|$ in a material. Moderate experimental requirements, straightforward data evaluation procedures and availability of powerful software $[6,2]$ make the powder PDF a perfect tool for routine investigations of local structures, provided that the problem at hand does not exceed a certain complexity. Limitations are mainly due to the powder specific angular projection of the PDF densities, which makes interatomic vectors of similar length indistinguishable even if their spatial orientations differ significantly. Furthermore, the frequency of powder PDF peaks increases with $|\mathbf{r}|^{2}$ and therefore problems quickly become unmanageable if the interatomic vectors of interest are getting long.

PDFs from single crystals $(3 D-P D F)$ may be calculated as the Fourier transform either of the total single crystal diffraction pattern (total $3 D-P D F$ ) or of the diffuse scattering alone $(3 D-\triangle P D F)$. In general, 3D-PDF investigations are experimentally more challenging than powder experiments, because high quality three-dimensional diffraction data sets need to be collected. On the other hand most of

* Correspondence author (e-mail: thomas.weber@mat.ethz.ch) the problems inherent to the powder PDF method may be overcome: the full 3D information about interatomic vectors is preserved and the peak frequency is approximately constant as a function of $|\mathbf{r}|$. In the case of $3 D-\triangle P D F s$ the number of significant peaks per volume even decreases with increasing distances. 3D-PDF methods were successfully applied to a series of disorder problems that are difficult to tackle with traditional methods like Monte Carlo modeling. In particular disorder in quasicrystals was studied with 3DPDF methods. Investigations cover phononic and phasonic disorder in an $\mathrm{Al}_{70} \mathrm{Co}_{12} \mathrm{Ni}_{18}$ decagonal quasicrystal [3, 4], as well as temperature dependent studies of structural disorder in the same compound [14]. The atomic structure of disordered clusters in the decagonal compound $\mathrm{Al}_{65} \mathrm{Cu}_{20} \mathrm{Co}_{15}$ was recently identified and refined in [9]. 3D-PDF methods are, however, by no means restricted to quasiperiodic compounds, but may equally well be applied to disordered periodic structures $[1,8]$.

This paper is intended to introduce the basic concepts and properties of the total $3 \mathrm{D}-\mathrm{PDF}$ and the $3 \mathrm{D}-\Delta \mathrm{PDF}$ methods. Application to real world examples is beyond the scope of this paper and will be presented in forthcoming publications.

\section{Theory}

\subsection{Definition}

In the following we will present the mathematical framework for the 3D-PDF theory. A similar approach was proposed e.g. in [16], which, however, focused on the description of diffuse scattering intensities. Here we will introduce a slightly different notation that more emphasizes total 3D-PDF and 3D- $\mathrm{PDF}$ aspects.

In analogy to the powder PDF method the total 3DPDF is defined as the Fourier transform of the total scattering from a single crystal:

$$
P_{\text {tot }}(\mathbf{x})=\mathrm{FT}\left\langle I_{\text {tot }}(\mathbf{h})\right\rangle \text {. }
$$

$P_{\text {tot }}(\mathbf{x})$ is also called the autocorrelation function of the real crystal, $I_{\text {tot }}(\mathbf{h})$ are the properly corrected total scattering intensities from a single crystal diffraction experiment, FT stands for the Fourier transformation and \langle\rangle denotes intensity averaging over time and over all coherently scat- 
tering volumes in a sample. In the following all vectors are expressed in dimensionless fractional lattice units, if not stated otherwise. For sake of simplicity we will ignore special experimental effects like anomalous, inelastic or multiple scattering and, without loss of generality, we will only use notations related to X-ray diffraction. The autocorrelation function of a crystal may be written as

$$
P_{\text {tot }}(\mathbf{x})=\sum_{M N}^{\text {cryst }} \varrho_{M}(\mathbf{x}) * \varrho_{N}(-\mathbf{x}) *\left\langle\delta\left(\mathbf{x}-\mathbf{x}_{M N}\right)\right\rangle .
$$

The double summation runs over all atoms in the crystal, $\varrho(\mathbf{x})$ is the electron density of an atom at the origin of the crystal's coordinate system, $\mathbf{x}_{M N}$ is the vector between the atoms $M$ and $N, \delta(\mathbf{x})$ is the Dirac function and the asterisk symbolizes the convolution operation. This general expression is valid for crystalline and non-crystalline materials. Crystals are built of unit cells and have an average structure, so that we can write

$$
\begin{aligned}
P_{\text {tot }}(\mathbf{x})= & \sum_{M N}^{\text {cryst }} \varrho_{M}(\mathbf{x}) * \varrho_{N}(-\mathbf{x}) * \delta\left(\mathbf{x}-\mathbf{R}_{u v w}-\mathbf{r}_{M N}\right) \\
& *\left\langle\delta\left(\mathbf{x}-\mathbf{u}_{M N}\right)\right\rangle,
\end{aligned}
$$

where $\mathbf{R}_{u v w}$ is the lattice vector between the unit cells occupied by the atoms $M$ and $N$, and $\mathbf{u}_{M N}$ is the difference between the real vector $\mathbf{x}_{M N}$ and the average structure related vector $\left(\mathbf{R}_{u v w}+\mathbf{r}_{M N}\right)$. In a next step the double summation is no longer performed over all atoms in the crystal, but over all atomic sites ${ }^{1} m$ and $n$ within the average unit cell. We further average over all interatomic vectors connecting atoms that are occupying the sites $m$ and $n$ in unit cells separated by a same lattice vector $\mathbf{R}_{u v w}$. The expression for the total 3D-PDF then becomes

$$
\begin{aligned}
P_{\text {tot }}(\mathbf{x})= & \sum_{\mathbf{R}_{u v w}}^{\text {cryst cell }} \sum_{m n}^{m n} p_{u v w}^{m n} \varrho_{m}(\mathbf{x}) * \varrho_{n}(-\mathbf{x}) \\
& * \delta\left(\mathbf{x}-\mathbf{R}_{u v w}-\mathbf{r}_{m n}\right) *\left\langle\delta\left(\mathbf{x}-\mathbf{u}_{u v w}^{m n}\right)\right\rangle,
\end{aligned}
$$

where $p_{u v w}^{m n}$ is the joint probability to simultaneously find an atom at site $m$ in one unit cell and another atom at site $n$ in a unit cell separated by $\mathbf{R}_{u v w}$. The term $\left\langle\delta\left(\mathbf{x}-\mathbf{u}_{u v w}^{m n}\right)\right\rangle$ is the probability density distribution of the random variable $\mathbf{u}_{u v w}^{m n}$, which represents the difference between the real and the average distance of atoms occupying sites $m$ and $n$ that are $u v w$ unit cells apart. For a chemical understanding it is often more convenient to express the pair correlations in terms of conditional probabilities, which are defined as $p_{u v w}^{m n}=c_{m} p_{u v w}(n \mid m)=c_{n} p_{\bar{u} \bar{w} \bar{w}}(m \mid n)$, where $c_{m}$ and $c_{n}$ are the average site occupation factors of atomic sites $m$ and $n$, respectively. The term $p_{u v w}(n \mid m)$ denotes the conditional probability that a site $n$ is occupied, provided that the $u v w$ unit cells separated site $m$ is also occupied. The definition of $p_{u v w}(m \mid n)$ is analogous.

\footnotetext{
1 An atomic site is defined as a placeholder for a specific element that occupies this position with a certain probability. The atom belonging to this site may rest on its ideal position or it may be displaced following a distribution function. It is important to note that in the case of substitutional disorder an atomic site needs to be defined for each of the elements that share an average position. The total number of atomic sites per unit cell therefore equals to the length of the atom list required for defining an average structure, if the symmetry of the crystal is represented in space group $P 1$.
}

The Fourier transform of the Bragg scattering is wellknown as the Patterson function, which is the autocorrelation function of the average structure of a crystal. It can be described by averaging Eq. (4) over all lattice vectors. As a consequence, each atomic site $m$ will be filled with a probability $c_{m}$, even if the results would lead to unphysical interatomic vectors. The joint occupational probability of every pair of average atoms is equal to the product of the occupancies of the individual atoms, i.e. $p_{u v w}^{m n}=c_{m} c_{n}$ and the distributions $\mathbf{u}_{u v w}^{m n}$ will split into two independent distributions of $\mathbf{u}_{m}$ and $\mathbf{u}_{n}$. The full formula for describing the Patterson function is

$$
\begin{aligned}
P_{\text {Pat }}(\mathbf{x})= & \sum_{\mathbf{R}_{u w w}}^{\text {cryst cell }} \sum_{m n} c_{m} c_{n} \varrho_{m}(\mathbf{x}) * \varrho_{n}(-\mathbf{x}) \\
& * \delta\left(\mathbf{x}-\mathbf{R}_{u v w}-\mathbf{r}_{m n}\right) *\left\langle\delta\left(\mathbf{x}-\mathbf{u}_{m}\right)\right\rangle *\left\langle\delta\left(\mathbf{x}-\mathbf{u}_{n}\right)\right\rangle .
\end{aligned}
$$

Typically, the average structure is well known before the real structure gets investigated. For a better understanding of local order phenomena it is therefore favorable to focus on the deviations from the average structure, i.e. to analyze $P_{\Delta}(\mathbf{x})=P_{\text {tot }}(\mathbf{x})-P_{\text {Pat }}(\mathbf{x})$. In reciprocal space this quantity is represented by the diffuse scattering $I_{\text {dif }}(\mathbf{h})=I_{\text {tot }}(\mathbf{h})-I_{\text {Bragg }}(\mathbf{h})$. The difference between Eqs. (4) and (5) yields:

$$
\begin{aligned}
P_{\Delta}(\mathbf{x})= & \sum_{\mathbf{R}_{u v w}}^{\text {cryst }} \sum_{m n}^{\text {cell }}\left[p_{u v w}^{m n}\left\langle\delta\left(\mathbf{x}-\mathbf{u}_{u v w}^{m n}\right)\right\rangle\right. \\
& \left.\quad-c_{m} c_{n}\left\langle\delta\left(\mathbf{x}-\mathbf{u}_{m}\right)\right\rangle *\left\langle\delta\left(\mathbf{x}-\mathbf{u}_{n}\right)\right\rangle\right] \\
& * \varrho_{m}(\mathbf{x}) * \varrho_{n}(-\mathbf{x}) * \delta\left(\mathbf{x}-\mathbf{R}_{u v w}-\mathbf{r}_{m n}\right) .
\end{aligned}
$$

The corresponding diffuse scattering intensity is the Fourier transform of Eq. (6):

$$
\begin{aligned}
I_{\mathrm{dif}}(\mathbf{h})= & \sum_{\mathbf{R}_{u v w}}^{\text {cryst }} \sum_{m n}^{\text {cell }}\left[p_{u v w}^{m n}\left\langle\exp \left(2 \pi i \mathbf{h} \mathbf{u}_{u v w}^{m n}\right)\right\rangle\right. \\
& \left.\quad-c_{m} c_{n}\left\langle\exp \left(2 \pi i \mathbf{h} \mathbf{u}_{m}\right)\right\rangle\left\langle\exp \left(2 \pi i \mathbf{h} \mathbf{u}_{n}\right)\right\rangle\right] \\
& f_{m}(\mathbf{h}) f_{n}^{*}(\mathbf{h}) \exp \left[2 \pi i \mathbf{h}\left(\mathbf{R}_{u v w}+\mathbf{r}_{m n}\right)\right] .
\end{aligned}
$$

In cases where the expressions in angle brackets may be approximated by Gaussians we can write $\langle\exp (2 \pi i \mathbf{h u})\rangle=\exp \left[-2 \pi^{2}\left\langle(\mathbf{h u})^{2}\right\rangle\right]=\exp \left(-\mathbf{h}^{T} \beta \mathbf{h}\right) \quad($ see [10]), where $\beta$ is a matrix representing dimensionless atomic displacement parameters (ADPs). The components of $\beta$ are defined as $\beta_{i j}=2 \pi^{2} a_{i}^{*} a_{j}^{*} U_{i j}$, where $U_{i j}$ are the frequently used ADPs in units of (length) ${ }^{2}$ and $a_{i}^{*}$ and $a_{j}^{*}$ are the lengths of the $i$-th and $j$-th reciprocal space vectors. Care has to be taken, however, if substitutional and displacive disorder are correlated. In such cases the distribution of $\mathbf{u}_{u v w}^{m n}$ may have a non-zero average and we obtain $\left\langle\exp \left(2 \pi i \mathbf{h u} \mathbf{u}_{u v w}^{m n}\right)\right\rangle=\exp \left(-\mathbf{h}^{T} \beta_{u v w}^{m n} \mathbf{h}\right) \exp \left(2 \pi i \mathbf{h} \overline{\mathbf{u}}_{u v w}^{m n}\right)$. The variable $\overline{\mathbf{u}}_{u v w}^{m n}$ indicates presence or absence of a socalled size effect distortion, depending on whether it is zero or not (see Section 3.3 for an example). In the harmonic approximation we can write:

$$
\begin{aligned}
I_{\mathrm{dif}}(\mathbf{x})= & \sum_{\mathbf{R}_{u v w}}^{\text {cryst cell }} \sum_{m n}\left[p_{u v w}^{m n} \exp \left(-\mathbf{h}^{T} \beta_{u v w}^{m n} \mathbf{h}\right) \exp \left(2 \pi i \mathbf{h} \overline{\mathbf{u}}_{u v w}^{m n}\right)\right. \\
& \left.-c_{m} c_{n} \exp \left(-\mathbf{h}^{T}\left(\beta_{m}^{\text {aver }}+\beta_{n}^{\text {aver }}\right) \mathbf{h}\right)\right] \\
& \times f_{m}(\mathbf{h}) f_{n}^{*}(\mathbf{h}) \exp \left[2 \pi i \mathbf{h}\left(\mathbf{R}_{u v w}+\mathbf{r}_{m n}\right)\right]
\end{aligned}
$$


where the matrix $\beta_{u v w}^{m n}$ describes the joint probability displacement densities of atoms occupying sites $m$ and $n$ that are separated by an average vector $\left(\mathbf{R}_{u v w}+\mathbf{r}_{m n}\right)$, while $\beta_{m}^{\text {aver }}$ and $\beta_{n}^{\text {aver }}$ are the space and time averaged ADPs of the sites $m$ and $n$, respectively. After considering centrosymmetry and applying some simple mathematical manipulations Eq. (8) may be expressed without complex exponential terms as

$$
\begin{aligned}
I_{\text {dif }}(\mathbf{x})=\sum_{\mathbf{R}_{u w w}}^{\text {cryst cell }} \sum_{m n} & \left\{p_{u v w}^{m n} \exp \left(-\mathbf{h}^{T} \beta_{u v w}^{m n} \mathbf{h}\right)\right. \\
& \cos \left[2 \pi \mathbf{h}\left(\mathbf{R}_{u v w}+\mathbf{r}_{m n}+\overline{\mathbf{u}}_{u v w}^{m n}\right)\right] \\
& -c_{m} c_{n} \exp \left[-\mathbf{h}^{T}\left(\beta_{m}^{\text {aver }}+\beta_{n}^{\text {aver }}\right) \mathbf{h}\right] \\
& \left.\cos \left[2 \pi \mathbf{h}\left(\mathbf{R}_{u v w}+\mathbf{r}_{m n}\right)\right]\right\} f_{m}(\mathbf{h}) f_{n}^{*}(\mathbf{h}) .
\end{aligned}
$$

\subsection{Properties}

Both, the total 3D-PDF and the 3D- $\triangle \mathrm{PDF}$ are not periodic in the presence of disorder. The symmetry must therefore not be described by a space group, but by a Laue point group having the same symmetry as the total diffraction pattern or the diffuse scattering, respectively. Laue symmetry of Bragg and diffuse scattering are often identical, but they may differ in special cases $[18,13]$.

The 3D- $\triangle \mathrm{PDF}$ provides information about features of the real structure that are not represented by the average structure. Positive $3 \mathrm{D}-\Delta \mathrm{PDF}$ values mean that the probability of finding scattering densities separated by the corresponding vector is higher than in the average structure and negative values indicate lower probabilities.

Using the 3D- $\triangle \mathrm{PDF}$ for analyzing disorder instead of the total 3D-PDF is advantageous for several reasons.

- The number of peaks in PDF space gets smaller, because only interatomic vectors having different real and average structure properties contribute to the $3 \mathrm{D}-\triangle \mathrm{PDF}$. This excludes all atoms that are fully ordered to a good approximation as well as atomic pairs that are completely uncorrelated, e.g. because they are separated by large distances [8].

- With the elimination of Bragg intensities one also removes any associated systematic and statistical errors at the same time. This is expected to significantly enhance the quality of the local structure information, since the integral errors of Bragg intensities are often of a same magnitude or even stronger than the integral intensities of diffuse scattering.

- The higher contrast of 3D- $\triangle \mathrm{PDF}$ maps allows qualitative and semi-quantitative conclusions about the underlying disorder problem just by a visual inspection of the patterns $[8,14]$.

An important feature, which may be directly extracted from the $3 \mathrm{D}-\triangle \mathrm{PDF}$ pattern, is the correlation length of local order. It is measured from the longest significant 3D$\triangle \mathrm{PDF}$ vectors and provides the information how far and along which direction a local structure property may affect its environment.
The magnitudes of 3D- $\triangle \mathrm{PDF}$ peaks essentially depend on the strength of pair correlations, on the product of the scattering power of the corresponding atomic pairs, on the contrast between the average and the real structure and on the multiplicity of the interatomic vectors. In general, the highest multiplicity is found for 3D-PDF peaks separated by lattice vectors, as the multiplicity of such vectors equals to the number of atomic sites per unit cell.

A further feature of the $3 \mathrm{D}-\triangle \mathrm{PDF}$ is that its origin peak may be directly calculated from the known average structure. It represents all interatomic vectors with length zero, i.e. $m=n$ and $\mathbf{R}_{u v w}=\mathbf{r}_{m n}=\mathbf{0}$. Since any atom is perfectly ordered with respect to itself we can write: $p_{000}^{m m}=c_{m} p_{000}(m \mid m)=c_{m}$ and $\mathbf{u}_{000}^{m m}=\mathbf{0}$. The origin peak may thus be calculated as:

$$
\begin{aligned}
P_{\Delta}^{\text {origin }}(\mathbf{x})=\sum_{m}^{\text {cell }} & {\left[c_{m} \delta(\mathbf{x})-c_{m}^{2}\left\langle\delta\left(\mathbf{x}-\mathbf{u}_{m}\right)\right\rangle\right.} \\
& \left.*\left\langle\delta\left(\mathbf{x}-\mathbf{u}_{m}\right)\right\rangle\right] * \varrho_{m}(\mathbf{x}) * \varrho_{m}(-\mathbf{x}),
\end{aligned}
$$

i.e. all quantities required for calculating the $3 \mathrm{D}-\triangle \mathrm{PDF}$ origin peak are available from the average structure. This property provides a unique link between the average structure and the 3D- $\triangle \mathrm{PDF}$ map, which was lost after diffuse and Bragg scattering were separated. An important application is the determination of a proper scale factor for diffuse scattering and $P_{\Delta}(\mathbf{x})$ maps of a model (see below).

From Eqs. (6) and (8) we know that the $3 \mathrm{D}-\Delta \mathrm{PDF}$ and its corresponding diffuse scattering may be described by the same set of parameters and therefore both, reciprocal space and PDF space oriented modeling, are likewise feasible. According to Parseval's law the following identity holds in the case of discrete Fourier transforms: $\sum_{i=0}^{K-1}\left|P_{\Delta}^{\text {obs }}[i]-P_{\Delta}^{\text {calc }}[i]\right|^{2}=1 / K \sum_{j=0}^{K-1}\left|I_{\text {dif }}^{\text {obs }}[j]-I_{\text {dif }}^{\text {calc }}[j]\right|^{2}, \quad$ where $K$ equals to the number of grid points. Apart from this constant factor the unweighted $\chi^{2}$ values of the model are the same in PDF and reciprocal space and therefore leastsquares refinements are expected to lead to exactly the same results independently of the reference space. A major difference, however, is found in the ability for masking or weighting specific features. Experimental artifacts like beam-stop shadow, parasitic scattering, saturations etc. are easily masked in reciprocal space and statistical errors are also more easily considered if refinements are done against diffuse scattering data. Refinements in PDF space on the other hand allow selective modeling of structural features. It was shown in [9] that this feature may significantly reduce the complexity of a given problem.

\section{Fingerprints of local order phenomena}

In the following we will demonstrate the impact of some basic disorder models on the appearance of single crystal PDF maps. For sake of simplicity the example structure will be two-dimensional. Correspondingly, the resulting PDFs will be called 2D-PDF or 2D- $\triangle \mathrm{PDF}$. The findings and discussions, however, may be straightforwardly transferred to three-dimensional space. 


\subsection{Displacive disorder}

The model structure is a chess board like arrangement of atoms $A$ and $B$ in the (non-standard) plane group $c 4 m m$. The scattering power of $B$ is assumed to be twice as strong as the one of $A$ and the average composition of the compound is $A B$. For our numerical calculations we used the atomic form factors of $\mathrm{Al}$ and $\mathrm{Fe}$, respectively. The lattice constant $a$ is defined to be $5 \AA$. For the displacive disorder model we assume absence of any substitutional disorder and all displacements are defined to be harmonic and isotropic with $U_{\text {iso }}=0.05 \AA^{2}$, i.e. $\beta_{i j} \approx 0.0395$ if $i=j$, and zero in all other cases. The 2D- $\Delta \mathrm{PDF}$ map is most easily obtained as the Fourier transform of the diffuse scattering calculated from Eq. (8), which in the case of pure displacive disorder in a two-dimensional structure simplifies to:

$$
\begin{aligned}
I_{\mathrm{dif}}(\mathbf{h})= & \sum_{\mathbf{R}_{u v}}^{\text {cryst cell }} \sum_{m n}\left[\exp \left(-\mathbf{h}^{T} \beta_{u v}^{m n} \mathbf{h}\right)-\exp \left(-\mathbf{h}^{T} 2 \beta^{\text {aver }} \mathbf{h}\right)\right] \\
& f_{m}(\mathbf{h}) f_{n}^{*}(\mathbf{h}) \cos \left[2 \pi \mathbf{h}\left(\mathbf{R}_{u v}+\mathbf{r}_{m n}\right)\right]
\end{aligned}
$$

In the first example all displacements are independent of each other, corresponding to the real structure that has the highest entropy compatible with the Bragg intensities. In such a case we obtain $\beta_{u v}^{m n}=2 \beta^{\text {aver }}$, except for $\beta_{00}^{m m}=\beta_{00}^{n n}=\mathbf{0}$, because a real atom is never displaced with respect to itself. Consequently, Eq. (11) further simplifies to

$$
I_{\text {dif }}(\mathbf{h})=\sum_{m}^{\text {cell }}\left[1-\exp \left(-\mathbf{h}^{T} 2 \beta^{\text {aver }} \mathbf{h}\right)\right]\left|f_{m}(\mathbf{h})\right|^{2} .
$$

The diffuse scattering and the corresponding PDF maps are shown in the top row of Fig. 1. Intensities are zero at the origin of reciprocal space and increase continuously with increasing distance. The decrease of the intensities at large vectors is caused by the atomic form factor. In the case of neutron experiments, where the atomic scattering power is constant as a function of reciprocal space coordinates, the diffuse scattering would converge to a maximum value at large scattering angles. The $2 \mathrm{D}-\triangle \mathrm{PDF}$ is obtained as the Fourier transform of the diffuse scattering and we observe a relatively narrow signal at the origin, which has a so-called Mexican hat shape: high densities at the origin are enclosed by a ring of negative densities. The integral of the $2 \mathrm{D}-\triangle \mathrm{PDF}$ is zero as it is true for any other strictly displacive disorder model. At larger distances there are no signals visible because the displacements are uncorrelated and cannot be distinguished from the average correlations. The Mexican hat profile of the peak can be explained by the fact that the distance of an atom to itself is always zero. Therefore, a distance of zero is more likely to be observed in the real than in the average structure. On the other hand any distance of an atom to itself having a length larger than zero is never present in a real structure, giving rise to negative $\triangle \mathrm{PDF}$ intensities around the positive peak at the origin. The observation that the positive peak is broader than a $\delta$-function, as it would be expected from our argumentation, is due to the fact that electron densities of the atoms have non-zero finite widths, which broaden the PDF signals.
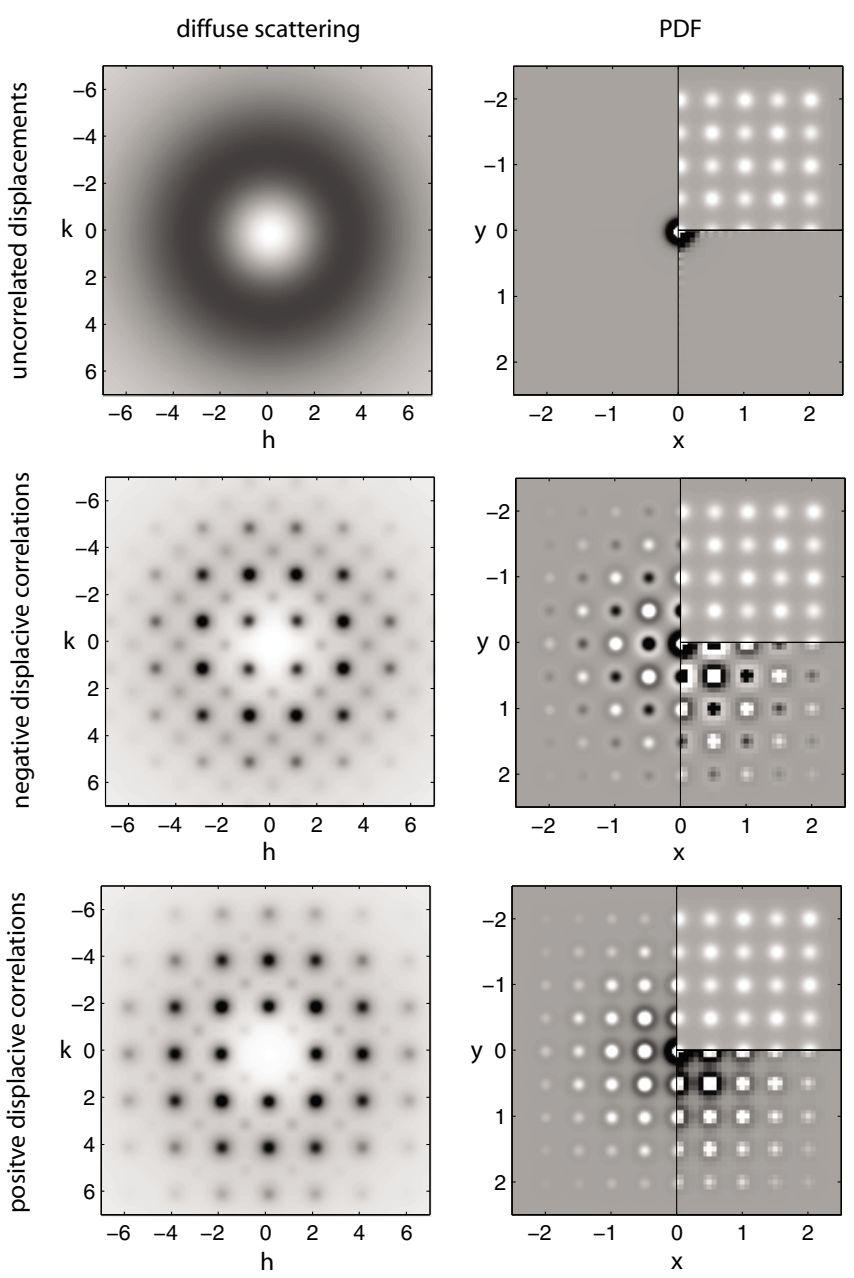

Fig. 1. Diffuse scattering and $2 \mathrm{D}-(\Delta) \mathrm{PDF}$ patterns of the displacive disorder models described in the text. Representations of diffuse and PDF maps follow conventional grey-scale schemes. In the case of diffuse scattering zero intensities are white, while strong intensities are shown in black. In the PDF space negative densities are shown in black, positive values are white and the zero level is in medium grey. On the left hand side of the PDF maps 2D- $\triangle$ PDFs are shown in very high resolution, while the lower right quarter shows the $2 \mathrm{D}-\triangle \mathrm{PDF}$ in resolutions corresponding to the visible diffuse scattering in the left column, i.e. in a real space resolution of about $0.7 \AA$. The upper right inset shows the total 2D-PDF pattern, i.e. the Fourier transform of Bragg and diffuse scattering. The total 2D-PDF and the 2D- $\triangle P D F$ color schemes are on different scales. In reality the total 2D-PDF intensities are by far stronger than the $2 \mathrm{D}-\triangle \mathrm{PDF}$. The Bragg reflections are not shown in the diffraction patterns. According to the $c$ centered lattice $(a=5 \AA)$ they would be visible at $h+k=2 n$.

In a next step we allow negative correlations between the displacements, i.e. next neighboring atoms tend to move along anti-parallel directions. The diffuse intensity of this model is described by Eq. (11). In our example the values of $\beta_{u v}^{m n}$ are defined by the equation

$$
\beta_{u v}^{m n}=2 \beta^{\text {aver }}\left[1-\sigma_{m n} \exp \left(-\left|\mathbf{R}_{u v}+r_{m n}\right|\right)\right],
$$

where $\sigma_{m n}=+1$ if $m$ and $n$ are occupied by same elements, otherwise it equals -1 . Any other parameters required in Eq. (11) are available from the average structure.

The resulting diffuse scattering and PDF patterns are shown in the middle row of Fig. 1. The diffuse scattering condenses around the Bragg positions and the envelope of the strong diffuse maxima is very similar to the diffuse scattering from the uncorrelated model. In 2D- $\triangle \mathrm{PDF}$ 
space the origin peak is exactly the same as for uncorrelated displacements, because this peak is exclusively defined by the average structure. Additional peaks are found at positions corresponding to the average interatomic vectors. As a consequence of the negative correlations of next neighboring atoms, different elements tend to move along opposite directions, while same elements prefer in-phase displacements. Consequently, real vectors between same elements have a high probability to be as long as the average vectors, leading to positive Mexican hat profiles. Different elements tend to avoid the average interatomic distances. Therefore $\triangle \mathrm{PDF}$ intensities are negative at such positions and the surroundings are positive, i.e. the corresponding peaks look like negative Mexican hats. Because of decreasing correlations, real and average displacements of atomic pairs assimilate with increasing distances and the magnitudes of the $\triangle \mathrm{PDF}$ peaks decrease accordingly.

The model for positive displacive correlations is analogously defined as the negative correlation model, but $\sigma_{m n}$ is +1 in all cases. The results are shown in the lower row of Fig. 1. The diffuse scattering pattern is similar to the negative correlation pattern, however, the weak diffuse peaks in the former case become strong and vice versa. In $2 \mathrm{D}-\Delta \mathrm{PDF}$ space all peaks are forming positive Mexican hat patterns, because all atomic pairs with shorter distances than the correlation length tend to move in phase. Any other properties are the same as before.

In theory observation of a Mexican hat profile is a clear indication of displacive disorder. In practice, however, truncation effects may also generate similar features, if the experimental diffuse scattering considered for calculating PDF maps has a circular or a spherical envelope. Careful examinations of the origin of Mexican hat style features is therefore required.

\subsection{Substitutional disorder}

The average structure of the substitutional disorder model is defined such that each site of the previous example is mixed occupied by $0.5 A$ and $0.5 B$ atoms. Atoms are resting at their average positions so that all displacement vectors $\mathbf{u}$ are zero. As a consequence of disorder the symmetry independent Wyckoff positions of the $5 \AA$ structure can no longer be distinguished and the lattice transforms from a $c$-centered to a primitive cubic lattice with $a=2.5 \AA$. The plane group symmetry becomes $p 4 m m$. The 2D$\triangle \mathrm{PDF}$ space of such a model can be described as:

$$
\begin{aligned}
P_{\Delta}(\mathbf{x})= & \sum_{\mathbf{R}_{u v}}^{\text {cryst }} \sum_{m n}^{\text {cell }}\left(p_{u v}^{m n}-0.25\right) \varrho_{m}(\mathbf{x}) * \varrho_{n}(-\mathbf{x}) \\
& * \delta\left(\mathbf{x}-\mathbf{R}_{u v}-\mathbf{r}_{m n}\right)
\end{aligned}
$$

and the diffuse scattering expression becomes:

$$
\begin{aligned}
I_{\mathrm{dif}}(\mathbf{h})= & \sum_{\mathbf{R}_{u v}}^{\text {cryst }} \sum_{m n}^{\text {cell }}\left(p_{u v}^{m n}-0.25\right) f_{m}(\mathbf{h}) f_{n}^{*}(\mathbf{h}) \\
& \times \cos \left[2 \pi \mathbf{h}\left(\mathbf{R}_{u v}+\mathbf{r}_{m n}\right)\right] .
\end{aligned}
$$

In the case of randomly distributed $A$ and $B$ atoms any pairs connected by a vector longer than zero have the joint occupational probabilities $p_{u v}^{A B}=p_{u v}^{B A}=p_{u v}^{A A}=p_{u v}^{B B}=c_{A} c_{B}=0.25$ and thus all of the corresponding terms in the summations of Eqs. (14) and (15) become zero. The conditional probabilities for the zeroth neighbor are $p_{00}(A \mid A)=p_{00}(B \mid B)=1$ and $p_{00}(B \mid A)=p_{00}(A \mid B)=0$. Consequently, $p_{00}^{m n}$ equals to 0.5 for $m=n$ and to zero otherwise. This simplifies Eq. (14) to

$$
\begin{array}{r}
P_{\Delta}(\mathbf{x})=0.25\left[\varrho_{A}(\mathbf{x}) * \varrho_{A}(-\mathbf{x})+\varrho_{B}(\mathbf{x}) * \varrho_{B}(-\mathbf{x})\right. \\
\left.-\varrho_{B}(\mathbf{x}) * \varrho_{A}(-\mathbf{x})-\varrho_{A}(\mathbf{x}) * \varrho_{B}(-\mathbf{x})\right] \\
=0.25\left[\varrho_{A}(\mathbf{x})-\varrho_{B}(\mathbf{x})\right] *\left[\varrho_{A}(-\mathbf{x})-\varrho_{B}(-\mathbf{x})\right],
\end{array}
$$

i.e. there is a single peak at the origin of PDF space, which is just the autocorrelation function of the difference between the electron densities of atoms $A$ and $\mathrm{B}$. The diffuse scattering obtained from such a model is proportional to the squared difference between the atomic form factors: $I_{\text {dif }}(\mathbf{h})=0.25\left|f_{A}(\mathbf{h})-f_{B}(\mathbf{h})\right|^{2}$. Diffuse scattering and PDF maps of this model are shown in the top row of Fig. 2.

In a next step occupational short-range order is introduced. In our first model, chess board like arrangements
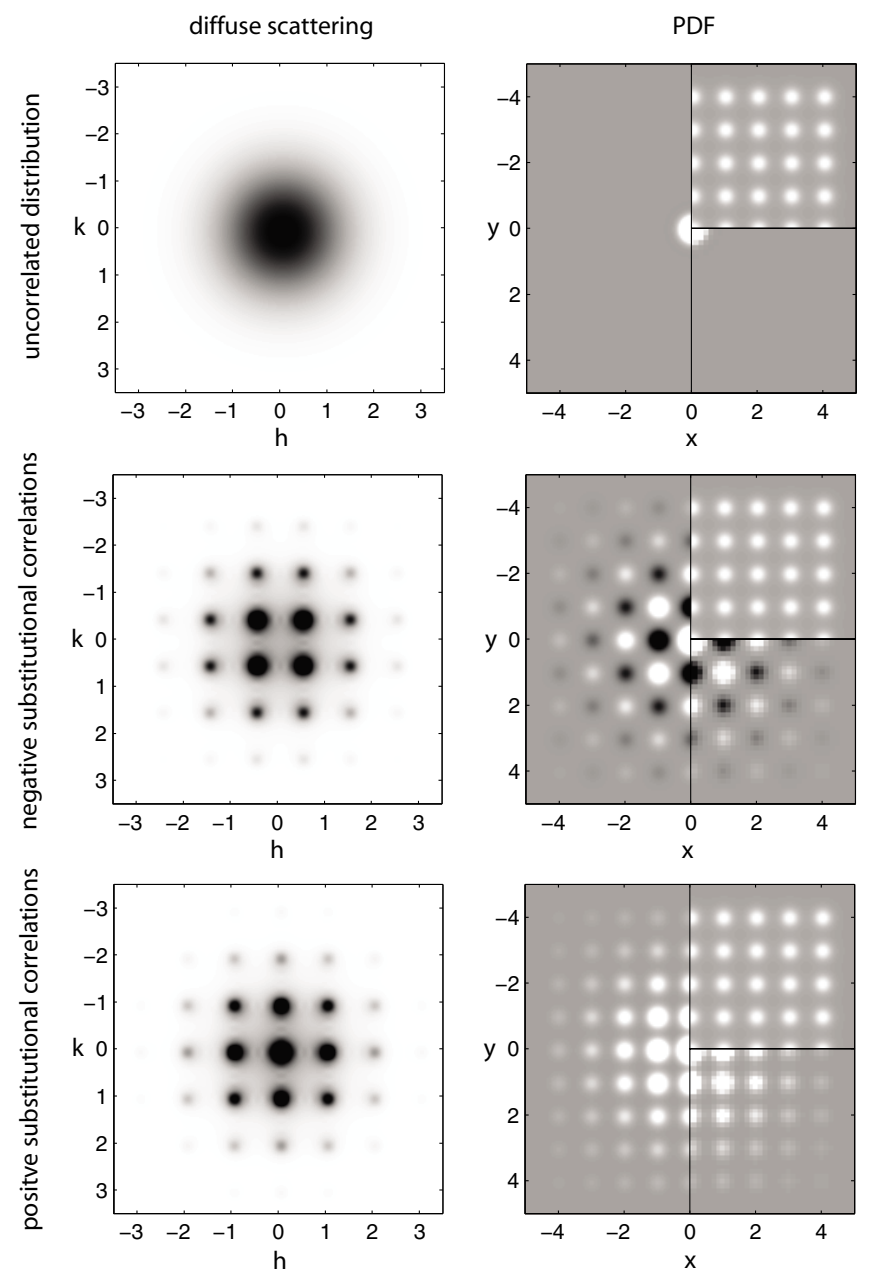

Fig. 2. Diffuse scattering and $2 \mathrm{D}-(\Delta) \mathrm{PDF}$ patterns of the substitutional disorder models described in the text. The grey-scale schemes and the meanings of sections in the PDF maps are as defined in Fig. 1. In reciprocal space the Bragg reflections are not shown. According to the $p$-lattice $(a=2.5 \AA$ ) they would be visible at integer $h, k$ positions. 
are formed on a local scale, i.e. there is a preference for hetero-atomic pairs $A B$ or $B A$ in the case that interatomic vectors $\mathbf{r}=u \mathbf{a}+v \mathbf{b}$ fulfill the condition $u+v=$ odd (based on the $2.5 \AA$ sized average unit cell), otherwise homo-atomic pairs $A A$ or $B B$ are more likely. The longer the interatomic vectors become the weaker the pair correlations get. For very long vectors the joint probabilities converge to $p_{u v}^{A A} \approx p_{u v}^{A B} \approx p_{u v}^{B A} \approx p_{u v}^{B B} \approx 0.25$. For any given distance $d=\sqrt{u^{2}+v^{2}}$ we obtain the relations $p_{d}(A \mid B)=p_{d}(B \mid A)=1-p_{d}(A \mid A)=1-p_{d}(B \mid B)$. In our model, an isotropic correlation field is defined as

$$
p_{d}(A \mid A)=0.5\left[1+\sigma_{u v} \exp (-d)\right],
$$

where $\sigma_{u v}=+1$ if $u+v=$ even, otherwise $\sigma_{u v}=-1$. The corresponding diffuse scattering and PDF patterns are seen in the middle row of Fig. 2. The diffuse scattering pattern shows broad maxima centered at positions $h, k=n / 2 \quad(n=$ odd $)$, i.e. at positions where the Bragg reflections are extinct due to the transformation of the $5 \AA$ sized $c$-lattice of the displacive disorder model discussed above to the $2.5 \AA p$-lattice in the present example. This behavior can be easily understood as the structure consists of domains having a local chess board structure similar to the displacive disorder model. The $2 \mathrm{D}-\triangle \mathrm{PDF}$ pattern, however, allows a more direct interpretation of the experimental results. Positive peaks are visible at positions $u+v=$ even, while peaks are negative at $u+v=$ odd, i.e. the sign of the 2D- $\Delta \mathrm{PDF}$ peaks directly indicates the properties of $\sigma_{u v}$. Again, the origin peak is the same as in the example of uncorrelated disorder. The profiles of the $\triangle \mathrm{PDF}$ peaks are constant, but as a consequence of decreasing correlations the integral densities of the peaks decrease with increasing distances. The decay of the densities directly reflects the properties of the exponential term in Eq. (17).

The bottom row of Fig. 2 shows the case of positive correlations where the atoms prefer being next to a same element. The definition of the disorder model is the same as for negative correlations, however, $\sigma_{u v}$ equals to +1 in all cases. Looking at the diffraction pattern we find that the diffuse peaks have moved to the Bragg positions, because the translation vector in the finite ordered domains of the real structure is the same as the average periodicity, i.e. $2.5 \AA$. In $2 \mathrm{D}-\triangle \mathrm{PDF}$ space the information obtained is again more directly related to the real structure properties: all peaks are positive, what directly reflects the definition of $\sigma_{u v}$. Any other properties like the width of the diffuse peaks and the decay of pair correlations are equivalent to the negative correlation patterns.

\subsection{Size effect distortions}

Displacive disorder stimulated by substitutional or occupational disorder is called a size effect. The corresponding model discussed in this section is defined as follows: $A$ and $B$ atoms are randomly distributed among the sites of the $2.5 \AA$ structure and the average displacements are equivalent to the displacive disorder model defined above. In addition the local interatomic distances are depending on the neighboring elements. For this model Eq. (8) may be rewritten as

$$
\begin{gathered}
I_{\text {dif }}(\mathbf{x})=\sum_{\mathbf{R}_{u v}}^{\text {cryst cell }} \sum_{m n}\left[p_{u v}^{m n} \exp \left(-\mathbf{h}^{T} \beta_{u v}^{m n} \mathbf{h}\right) \exp \left(2 \pi i \mathbf{h} \overline{\mathbf{u}}_{u v}^{m n}\right)\right. \\
\left.-0.25 \exp \left(-\mathbf{h}^{T} 2 \beta^{\mathrm{aver}} \mathbf{h}\right)\right] \\
f_{m}(\mathbf{h}) f_{n}^{*}(\mathbf{h}) \exp \left[2 \pi i \mathbf{h}\left(\mathbf{R}_{u v}+\mathbf{r}_{m n}\right)\right] .
\end{gathered}
$$

$A B$ distances are the same as in the average structure, while $A A$ distances are shorter and $B B$ pairs are further apart. In addition, we assume that the size effect induced shifts are much smaller than the independent displacements, which may be coming e.g. from thermal vibrations. This assumption allows us to further accept a harmonic approximation for the average displacements. From the definition of our model it follows that the disorder parameters $p_{u v}^{m n}$ and $\beta_{u v}^{m n}$ are exactly the same as in the cases of uncorrelated disorder discussed above, i.e. $p_{00}^{A A}=p_{00}^{B B}=0.5, p_{00}^{A B}=p_{00}^{B A}=0.0$ and $p_{u v}^{m n}=0.25$ for any non-zero vector. The joint displacement probabilities follow the relation $\beta_{u v}^{m n}=2 \beta^{\text {aver }}$, except for $\beta_{00}^{m m}=\beta_{00}^{n n}=\mathbf{0}$. The only free model parameter is $\overline{\mathbf{u}}_{u v}^{m n}$. It is defined such that $A A$ pairs neighboring along a main axis, are shifted by $0.01 \AA$ along this direction and $B B$ distances are $0.01 \AA$ shorter. The size effect shifts decrease by a half for each additional step, i.e. it is $0.005 \AA$ for the second next neighbor, $0.0025 \AA$ for the third, and so on. For sake of simplicity we assume that size effect displacements are only effective for elements neighboring along $\langle 100\rangle$ directions.

The resulting diffuse scattering and PDF maps are seen in Fig. 3. The diffuse scattering shows asymmetries with respect to integer $h, k$ values, which is a very typical pattern for size-effect distortions [18]. The 2D- $\Delta$ PDF pattern straightforwardly reflects the disorder model. Correlations are only visible along the main axes. Similar to reciprocal space the size effect is seen in strong asymmetries along radial directions. For the present example the interatomic vectors point to positive values if the distances are larger than the average distances and negative for shorter separations. This observations can be directly interpreted such that distances between pairs of strong scatterers are longer than between pairs of weak scatterers.
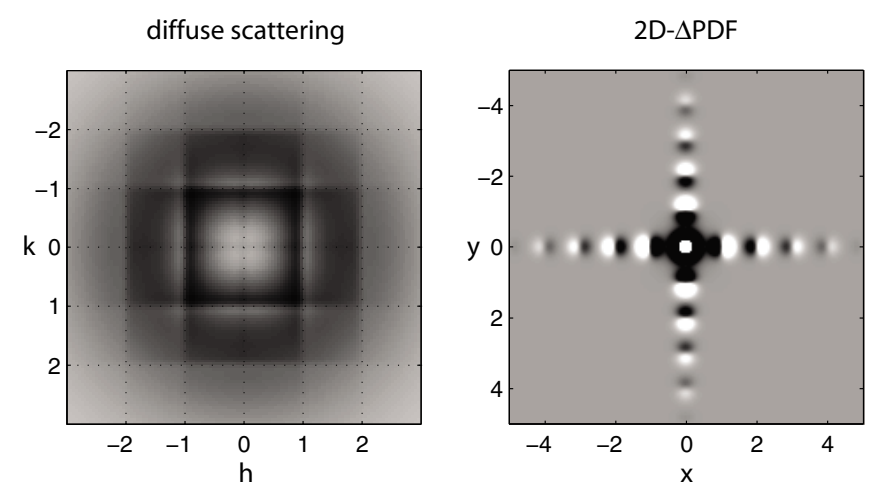

Fig. 3. Diffuse scattering and $2 \mathrm{D}-\Delta \mathrm{PDF}$ patterns of the size effect disorder model described in the text. The grey-scale schemes are as defined in Fig. 1. Bragg reflections are not shown, but would be visible at integer $h, k$ positions according to the $p$-lattice $(a=2.5 \AA)$. The dotted grid in the diffuse scattering pattern is at integer $h$ and $k$ values to demonstrate the asymmetry of the diffuse intensities with respect to the integer Bragg positions. 


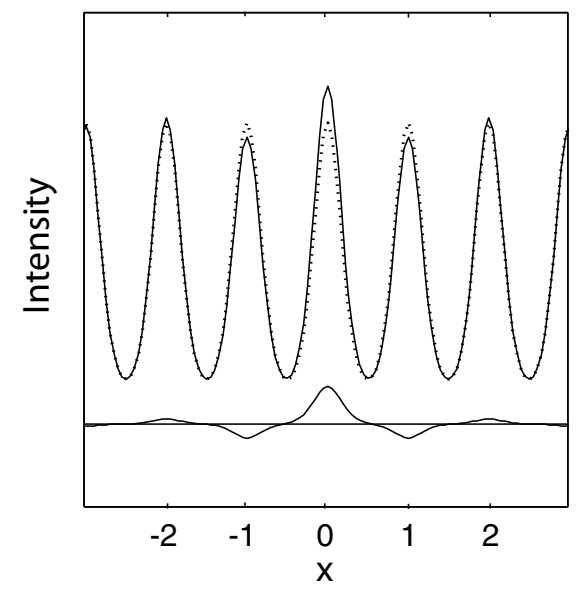

Fig. 4. Profiles from the intensities of the total 2D-PDF (upper full line), of the 2D- $\triangle \mathrm{PDF}$ (lower full line) and of the Patterson function (dotted line) corresponding to the negative substitutional correlation model shown in Fig. 2 middle row. The scans are taken at $y=0$. All PDF densities are on the same scale. The horizontal line represents the zero density level. It is clearly seen that the Patterson function, which was calculated as the difference between the total 2D-PDF and the 2D- $\triangle \mathrm{PDF}$, heavily dominates the total PDF.

\subsection{Full PDF vs. $\Delta$ PDF modeling}

The total 2D-PDF patterns of the displacive and substitutional disorder models discussed above are seen in the upper right quarters of the PDF maps shown in Figs. 1 and 2. Differences between the models are hardly visible. The reason for the similarity of the patterns is that the total 3D-PDF is heavily dominated by the Patterson function so that grey-scale representations do not allow any identification of particular disorder models. To demonstrate the relations between the full PDF, the $\triangle \mathrm{PDF}$ and the Patterson function more clearly one-dimensional scans are shown in Fig. 4. The data are based on the negatively correlated substitutional disorder model discussed above, which was selected because it shows the strongest contrast between the real and the average structure. The dominance of the Patterson function is clearly visible and nicely demonstrates the advantage of the $\triangle \mathrm{PDF}$ over the total PDF method. This example also emphasizes a significant advantage of the single crystal PDF method over the powder PDF, where a proper separation of Bragg and diffuse scattering is by far more complicated than in single crystal diffraction patterns.

\section{Pathology and remedies}

In the following chapter we will discuss a few typical experimental and modeling problems associated with the investigation of disorder and we will propose approaches for overcoming them. The examples are not exhaustive and the problems are not specific for the PDF method. The PDF space, however, is very well suited for a comprehensive understanding of the impact of corresponding errors on the real structure properties derived from any diffuse scattering studies, including Monte Carlo simulations.

\subsection{Reciprocal space resolution}

The experimental point-spread function in a diffuse scattering experiment is a convolution of various factors coming from the instrument or from the sample. In the following any experimental influence on Bragg reflection profiles will be called a resolution effect. With this definition Bragg peak profiles become a direct measure of the resolution function. Note that heavily strained samples and paracrystals are excluded from our discussion, because the Bragg peaks are broadened by disorder and therefore the Bragg peak profiles do not exclusively represent the experimental resolution function. Note also that the determination of the average structure is usually not affected by the resolution function, because it does not influence the integral Bragg peak intensities. Resolution function determining factors include e.g. beam divergence, spectral bandwidth of the beam, vibrations of the crystal or the instrument, cross-section of the beam with the sample, point spread function of the detector, step width in the data collection scans, artifacts in reciprocal space reconstructions and mosaicity of the sample. The complexity of the problem makes straightforward solutions, as e.g. routinely done in Rietveld powder refinements, difficult. To demonstrate the impact of the various effects on the single crystal PDF properties we roughly divided the resolution effects into three categories.

First, a function is considered that broadens Bragg peaks isotropically and uniformly. To a first approximation, most of the effects mentioned above belong to this class. Mathematically, this effect can be described as a convolution of a perfect diffraction pattern with a constant profile function. According to the convolution theorem the effect in 3D-PDF space is a multiplication of the true PDF with the Fourier transform of the resolution function. If we assume a Gaussian as a peak-shape function then its Fourier transform is also a Gaussian. The larger a PDF vector gets the more attenuated become the observed PDF densities as a consequence of the resolution effect. Profiles of the PDF peaks are (practically) not affected. The effect is shown in Fig. 5, second row. Typical half widths of Bragg reflections measured in synchrotron radiation experiments are in the order of $10^{-3} \AA^{-1}$ (reciprocal space units are defined as $\left.d^{*}=2 \sin (\theta) / \lambda\right)$ ). In such cases the true PDF intensities get damped by about $3.5 \%$ at a distance of $100 \AA$ and by $50 \%$ at about $440 \AA$ [15], i.e. only long vectors are seriously affected. In typical in-house experiments the reciprocal space resolution is in the order of $10^{-2} \AA^{-1}$ and thus significant effects are expected at about one order of magnitude shorter distances. Resolution effects can be corrected in PDF space by dividing the observed PDF maps with the Fourier transform of the resolution function. In general, no corrections are required if the diffuse scattering features are by far broader than the resolution function.

The other two effects considered are causing broadenings of the peaks along radial or angular directions. The resolution function is not constant, but increases linearly with increasing distances from the origin of reciprocal space. Radial resolution effects are usually coming from the spectral width of the primary beam, while angular 
broadenings may be caused e.g. by the mosaicity of the sample, by vibrations or by the data collection step width. As seen in the third and fourth row of Fig. 5 the effects are analogous in reciprocal and PDF space. In the case of radially shaped resolution functions Bragg's law transforms the constant monochromator's bandwidth $\Delta \lambda / \lambda$ to a constant $\Delta d / d$ in PDF space leading to the radial broadenings that are increasing with increasing distances. The angular resolution effects are also directly transformed from one space to the other, since rotations of the coordinate systems are covariant in real and reciprocal space.

There is no easy correction for such angular or radial resolution effects, but the resolution function may be simulated and applied to PDF or diffuse scattering model maps by using numerical methods. If not considered in
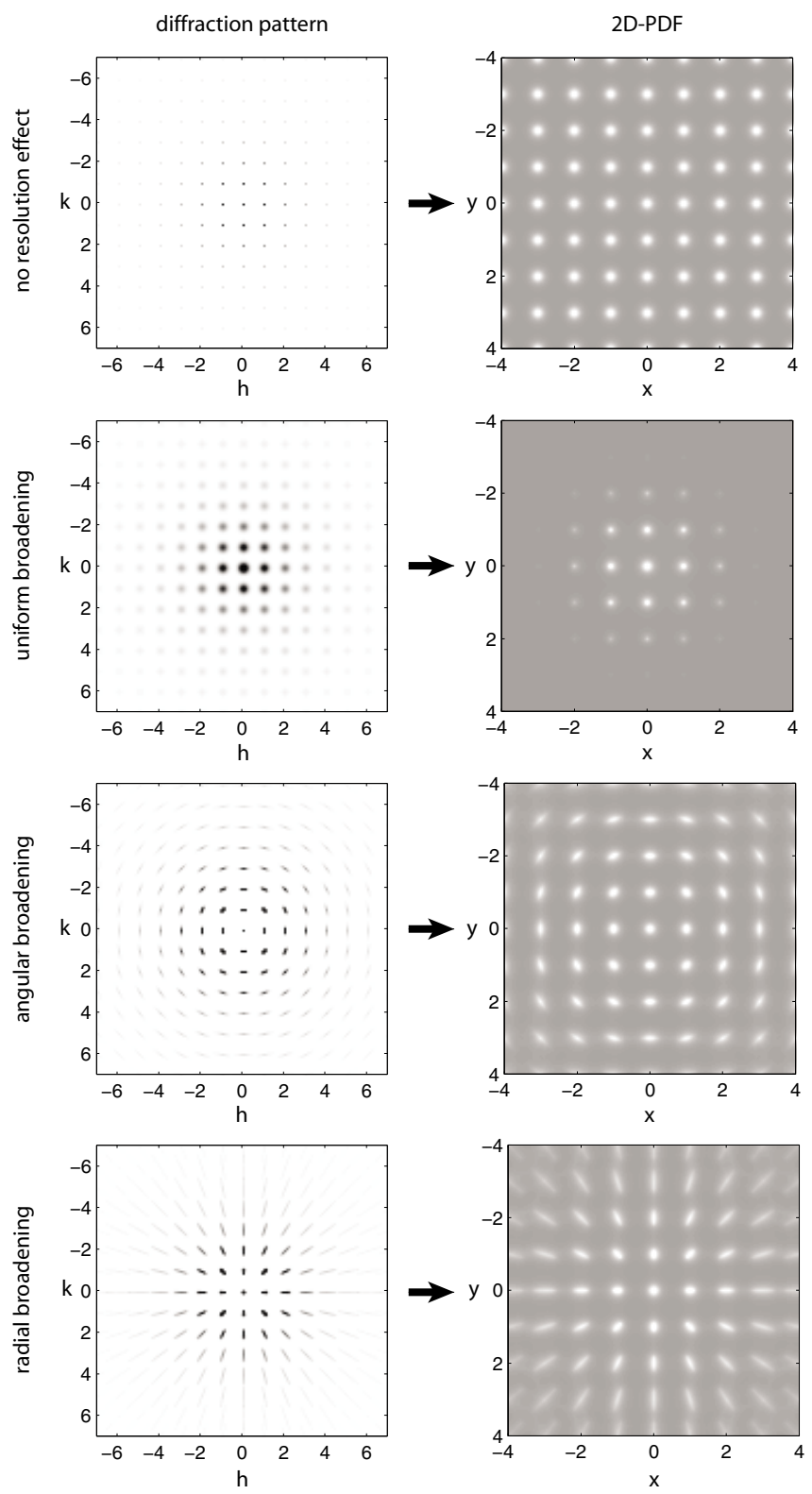

Fig. 5. Impact of reciprocal space resolution effects on the PDF pattern as described in the text. The top row shows a reference model of a diffraction experiment that is free of resolution effects. For the sake of clarity the examples are showing the resolution effects for Bragg scattering and for the Patterson function only, but the results may be straightforwardly transferred to diffuse scattering experiments. The grey-scale schemes are as defined in Fig 1. diffuse scattering studies local atomic displacements will get overestimated independent of the modeling technique applied. Details will be discussed in forthcoming papers.

\subsection{Separation of Bragg and diffuse data}

In many cases separation of Bragg and diffuse scattering is straightforward, because the signals are found in different layers or lines. In other cases diffuse scattering is by far broader than the Bragg profiles and interpolation of the diffuse intensities beneath the Bragg scattering provides a reasonable approximation to the real diffuse intensities. More difficult situations are found if narrow diffuse maxima are exactly at or very close to the Bragg positions. In such cases the true diffuse scattering can hardly be reconstructed without providing additional information like theoretical profile functions. In the following we will propose three possibilities for solving such problems.

First, a total 3D-PDF study would overcome the necessity of separating Bragg and diffuse data. However, this approach requires perfectly measured diffuse and Bragg data at the same time - a task that is very challenging but feasible [12]. Second, despite the fact that interpolations may provide only rough approximations to the true diffuse scattering, the diffuse scattering beneath the Bragg peaks may be reconstructed using the so-called punch-and-fill method as discussed in [3, 4]. The method uses a simple interpolation algorithm for filling the gaps after elimination of Bragg peaks. It was shown that the resulting 3D$\triangle \mathrm{PDF}$ patterns are reliable if only very short vectors are considered, however, long PDF vectors may get heavily biased by this approximation. Finally, optimization of a $3 \mathrm{D}-\triangle \mathrm{PDF}$ model could be done in reciprocal space by applying zero weights to the Bragg scattering affected regions as it is typically done in Monte Carlo refinements (e.g. $[17,7,11])$. The consequences for the results obtained are very similar to the punch-and-fill method. In the case that the masked diffuse peaks are very narrow their major Fourier components correspond to long vectors in PDF space. By suppressing such experimental results, the information about long $3 \mathrm{D}-\triangle \mathrm{PDF}$ vectors may no longer be well defined. Independent of the modeling technique used, any analysis of long distance correlations may therefore become unreliable, unless a correct model for the decay of correlations is enforced.

\subsection{Scale factor determination}

The determination of a proper scale factor for a $3 \mathrm{D}-\Delta \mathrm{PDF}$ model is not trivial after Bragg and diffuse scattering was separated. In particular in the case of dominant substitutional disorder, diffuse scattering and $3 \mathrm{D}-\triangle \mathrm{PDF}$ maps linearly depend on the pair correlation parameters $p_{u v w}^{n m}$, i.e. they fully correlate with the scale factor. A proper determination of the scale factor is therefore not possible without providing additional information. Fortunately, the origin peak of the $3 \mathrm{D}-\triangle \mathrm{PDF}$ map provides such a constraint. As mentioned above the origin peak is independent of the disorder model and it can be calculated from the disorder properties known from the average structure like ADPs and site occupation factors. The scale factor is easily ob- 
tained as the ratio of the experimental and calculated origin peaks. Any further pair correlation coefficients only need to be determined relative to the origin peak and thus the linear correlation between the scale factor the 3D$\triangle \mathrm{PDF}$ gets resolved.

There are, however, some practical problems that may bias a proper determination of the scale factor. It is a property of the Fourier transformation that the short $3 \mathrm{D}-\Delta \mathrm{PDF}$ vectors defining the origin peak are heavily affected by broad scattering intensities. A careful handling of background scattering is therefore essential as typical sources of background like electronic noise of the detector, air, fluorescence or Compton scattering as well as scattering from the crystal holder are smooth signals in most cases. A separation of background and diffuse scattering is easily done by interpolation, if the diffuse intensities are fully condensed in features that are narrow at least along one dimension like diffuse streaks or layers. In cases, where parts of the diffuse signal are very broad along all directions, diffuse scattering and background scattering are difficult to distinguish, and, as a consequence, the scale factor would get biased by the background. This problem can be minimized experimentally by using optimized setups and modern detectors like the PILATUS [5], which is not only free of intrinsic noise, but also allows suppression of florescence scattering. From a modeling point of view the problem can only be tackled, if additional constraints for pair correlations beyond the zeroth neighbor may be applied, as e.g. in the case of molecules, where a series of conditional occupational probabilities are often known to equal to unity. Such additional information allows extending the volume in PDF space that may be predicted from the average structure alone and make the scale factor determination significantly less sensitive to experimental artifacts.

\section{Conclusions}

Single crystal based PDF analysis is a straightforward method for analyzing disorder. Local order properties may be directly accessed through the Fourier transform of the full or the diffuse diffraction pattern. In the latter case the local order features are visible with higher contrast and they are less affected by experimental problems. A phase problem, as it is well-known for the determination of the average structure, does not exist, because the PDFs are directly calculated as the Fourier transform of the scattering intensities. Many local structure properties of a disordered material can be directly extracted from the single crystal PDF patterns just by a visual inspection. The 3D$(\Delta)$ PDF patters provides direct information about the type of disorder (e.g. displacive or substitutional disorder) present in a structure and about the local properties of the atoms involved, what is certainly the major advantage of PDF methods over reciprocal space oriented investigations. The formulas for calculating diffuse scattering or PDF maps from a PDF disorder model are perfectly suited for least-squares refinements. A computer program for performing such investigations is currently under development.
Powder and single crystal PDF methods are complementary. The major advantages of powder diffraction methods are that large single crystals are not required as well as the relatively simple and very fast experiments, which allow performing studies that cannot be done with single crystals. Single crystal PDF analysis on the other hand is to be preferred if complex local order needs to be investigated or if interatomic correlations become very long, even though collection of high quality diffuse scattering is much more complicated than in the case of powder experiments. For complex problems the by far better resolution of PDF peaks in three-dimensional space allows investigations of disorder problems that cannot be analyzed with powder diffraction techniques. The ability for elimination of average structure features and the possibility for selectively modeling parts of the disordered structure as demonstrated in [9], extends the tool-box for handling even extremely complex problems. Such advanced modeling techniques are much easier applied to single crystal PDFs than to powder diffraction data.

\section{References}

[1] M. H. Chao, K. D. M. Harris, B. M. Kariuki, C. L. Bauer, B. M. Foxman: Characterization of intermolecular interactions in a disordered solid via a one-dimensional Patterson synthesis. J. Phys. Chem. B 2002, 106, 4032.

[2] C. L. Farrow, P. Juhas, J. W. Liu, D. Bryndin, E. S. Bozin, J. Bloch, T. Proffen, S. J. L. Billinge: PDFfit2 and PDFgui: computer programs for studying nanostructure in crystals. J. Phys.: Condens. Matter 2007, 19, 335219.

[3] M. Kobas, T. Weber, W. Steurer: Structural disorder in the decagonal Al-Co-Ni. I. Patterson analysis of diffuse X-ray scattering data. Phys. Rev. B 2005, 71, 224205.

[4] M. Kobas, T. Weber, W. Steurer: Structural disorder in the decagonal Al-Co-Ni. II. Modelling. Phys. Rev. B 2005, 71, 224206.

[5] P. Kraft, A. Bergamaschi, Ch. Broennimann, R. Dinapoli, E. F. Eikenberry, B. Henrich, I. Johnson, A. Mozzanica, C. M. Schlepuetz, P. R. Willmott, B. Schmitt: Performance of single-photoncounting pilatus detector modules. J. Synchrotron Rad. 2009, 16, 368.

[6] T. Proffen, S. J. L. Billinge: PDFFIT, a program for full profile structural refinement of the atomic pair distribution function. $J$. Appl. Crystallogr. 1999, 32, 572.

[7] T. Proffen, T. R. Welberry: Analysis of diffuse scattering of single crystals using Monte Carlo methods. Phase Transit. 1998, 67, 373.

[8] P. Schaub, T. Weber, W. Steurer: Exploring local disorder in single crystals by means of the three-dimensional pair distribution function, Philosoph. Mag. 2007, 87, 2781.

[9] P. Schaub, T. Weber, W. Steurer: Analysis and modelling of structural disorder by the use of the three-dimensional pair distribution function method exemplified by the disordered twofold superstructure of decagonal $\mathrm{Al}-\mathrm{Cu}-\mathrm{Co}$. J. Appl. Crystallogr. 2011, 44, 134.

[10] K. N. Trueblood, H. B. Bürgi, H. Burzlaff, J. D. Dunitz, C. M. Gramaccioli, H. H. Schulz, U. Shmueli, S. C. Abrahams: Atomic displacement parameter nomenclature - report of a subcommittee on atomic displacement parameter nomenclature. Acta Crystallogr. 1996, A52, 770 .

[11] T. Weber, H. B. Bürgi: Determination and refinement of disordered crystal structures using evolutionary algorithms in combination with Monte Carlo methods. Acta Crystallogr 2002, A58, 526.

[12] T. Weber, S. Deloudi, M. Kobas, Y. Yokoyama, A. Inoue, W. Steurer: Reciprocal-space imaging of a real quasicrystal. a feasibility study with PILATUS 6M. J. Appl. Crystallogr. 2008, 41, 669. 
[13] T. Weber, M. A. Estermann, H. B. Bürgi: Structural complexity of a polar perhydrotriphenylene inclusion compound brought to light by synchrotron radiation. Acta Crystallogr. 2001, B57, 579 .

[14] T. Weber, M. Kobas, W. Steurer: The disordered 8 Angstrom superstructure of a decagonal $\mathrm{Al}_{70} \mathrm{Co}_{12} \mathrm{Ni}_{18}$ quasicrystal. Ferroelectrics 2004, 305, 213.

[15] T. Weber, W. Steurer: Structural Disorder in Quasicrystals. In: Diffuse Scattering and the Fundamental Properties of Materials (Eds. R. I. Barabash, G. E. Ice, P. E. A. Turchi), pp. 239-258.
[16] T. R. Welberry, B. D. Butler: Diffuse X-ray scattering from disordered crystals. Chem. Rev. 1995, 95, 2369.

[17] T. R. Welberry, T. Proffen, M. Bown. Analysis of single-crystal diffuse X-ray scattering via automatic refinement of a Monte Carlo model. Acta Crystallogr. 1998, A54, 661.

[18] T. R. Welberry, Diffuse X-ray Scattering and Models of Disorder, Oxford University Press, New York 2004. 